\title{
Galectin-3: clinical utility and prognostic value in patients with heart failure
}

This article was published in the following Dove Press journal:

Research Reports in Clinical Cardiology

5 February 2013

Number of times this article has been viewed

\section{Frank Kramer \\ Clinical Sciences, Global Biomarker Strategy and Development, Bayer HealthCare, Wuppertal, Germany}

\begin{abstract}
This review summarizes the key findings of 14 clinical trials in which Galectin-3 was assessed as a biomarker of heart failure. In addition, most relevant information available about Galectin-3 biology generated in in vitro and in vivo research is discussed in the context of heart failure.
\end{abstract}

Keywords: biomarker, cardiovascular disease, therapy monitoring, prediction of outcome, patient stratification

\section{Introduction}

Heart failure (HF) is a deleterious condition with an estimated prevalence of about $1 \%-2 \%$ and is responsible for about $1 \%-2 \%$ of all health care spending in the Western world. HF biomarkers are of increasing importance for both physicians and preclinical researchers. Quantification of biomarkers is a valuable tool in therapy monitoring, contributes to the prediction of clinical outcome, and can play a key role in patient stratification. This review summarizes the most relevant scientific knowledge that has accumulated so far on Galectin-3 (Gal-3) as a biomarker in HF.

First, the literature on the role of Gal-3 in different physiological and pathophysiological conditions beyond cardiovascular diseases is reviewed to give the reader a comprehensive overview of the biological function of the lectin. Following this, the most important findings made in Gal-3 knockout mice are summarized. Since the most recent review on the role of Gal-3 in HF was published several years ago and various additional clinical studies assessing its utility for the management of cardiovascular disease have been published since, an update on this topic is urgently needed. Finally, 14 clinical studies are summarized and the implications of their results assessed for clinical practice.

It is hoped that this review will provide the basis for the planning of further investigations of Gal-3 in future clinical trials in its summarizing of the most relevant current knowledge about this biomarker in the context of cardiovascular diseases.

\section{Molecular background and function of Gal-3}

Gal-3 (also known as Mac-2, CBP-35, L29, LBP, or eBP) belongs to the family of $\beta$-galactoside-binding proteins with a preference for lactose and $\mathrm{N}$-acetyllactosamine. ${ }^{1}$ The human LGALS3 gene, which is located on chromosome 14, codes for a 250-amino acid protein with a molecular weight of $29-36 \mathrm{kDa} .{ }^{2}$ Gal-3 binds to a wide array of extracellular matrix proteins such as tenascin, fibronectin, and laminin due to its 
carbohydrate recognition domain and collagen-like protein domains. There are a number of cell types that express Gal-3, including neutrophils, macrophages, mast cells, fibroblasts, and osteoclasts. Gal-3 has been detected in the lung, stomach, colon, uterus, and ovary. ${ }^{3}$ As Gal-3 lacks a signal sequence, which is essential for the classical secretory pathway, it is mainly found in the cytoplasm but can also be detected within the nucleus, as the first eleven amino acids serve as a nuclear localization domain. ${ }^{4}$ However, Gal-3 can be secreted via nonclassical pathways. ${ }^{5}$ Nuclear Gal-3 has the ability to alter gene expression through direct interaction with or stabilization of transcription factors and by regulation of nuclear pre-messenger (m) RNA splicing. ${ }^{6-8}$ A comprehensive overview of the biological functions of Gal-3 in the nucleus can be found elsewhere. ${ }^{9}$

In the cytosol, Gal-3 inhibits apoptosis, controls survival, and regulates exocytosis by interacting with a broad range of cytosolic proteins.

Though Gal-3 cannot be secreted via the classical endoplasmic reticulum-Golgi pathway, the lectin is abundant in different extracellular compartments, such as in serum, on cell surfaces, and in the extracellular matrix..$^{5,10,11}$ It is postulated that after a heat shock protein-mediated Gal-3 accumulation at the cytoplasmic side of the plasma membrane, the lectin is secreted in an ectocytotic process. ${ }^{12}$ This hypothesis is supported by the observation that dendritic cell-derived exosomes contained Gal-3 protein. ${ }^{13}$ It was suggested that enzymatic breakdown of the exosome by, for example, members of the phospholipase family, leads to the release of Gal-3 from its transporter vesicles. ${ }^{5}$

After secretion, Gal-3 exhibits multiple autocrine and paracrine properties. Gal-3 activates neutrophils, mast, and T-cells, regulates cell adhesion, induces apoptosis, and provides angiogenic signals. ${ }^{14-18}$ Depending on the cell type and the balance between intracellular and extracellular Gal-3 in a biological system, Gal-3 can inhibit or induce cell growth and differentiation. A comprehensive review of the literature regarding physiological properties of nuclear, cytolsolic, and extracellular Gal-3 is beyond the scope of this manuscript (see Dumic et al for further details ${ }^{19}$ ).

Besides its autocrine and paracrine properties, Gal-3 plays an important role in the defense against pathogens. Gal-3 enhances pro-inflammatory signals by being chemotactic to macrophages and monocytes, mediating adhesion of neutrophils, inducing release of pro-inflammatory factors from white blood cells and mast cells, and participating in the phagocytic clearance of apoptotic neutrophils by macrophages. ${ }^{20-23}$ Furthermore, Gal-3 directly interacts with galactoside-containing glycoconjugates on the surface of pathogens. It has been shown that Gal-3 recognizes and binds to Streptococcus pneumonia, Escherichia coli, Pseudomonas aeruginosa, Candida albicans, Mycobacterium tuberculosis, and different Salmonella strains. ${ }^{24-28}$ An overview about the molecular background and function of Gal-3 is displayed in Figure 1.

\section{Gal-3 in disease models}

In the myocardium, the Gal-3 expression level is almost undetectable in cardiomyocytes, whereas cardiac fibroblasts express higher levels of this carbohydrate-binding lectin. ${ }^{29}$ Recently, Schroen et al identified strong upregulation of Gal-3 mRNA in a rat model of renin-dependent hypertension. ${ }^{30}$ In further studies, Sharma et al found that myocardial Gal-3 was increased in those animals, which progress to $\mathrm{HF}^{29}$ They also found that infusion of Gal-3 into the pericardium induced myocardial collagen deposition and remodeling. A profibrotic effect of Gal-3 was also identified for hepatic fibrosis and myofibroblast differentiation and in a rat model treated by the naturally occurring anti-fibrotic peptide $\mathrm{N}$-acetyl-seryl-aspartyl-lysyl-proline. ${ }^{31,32}$ In addition, a very recent publication illustrates the role of Gal-3 as a mediator of aldosterone-induced vascular fibrosis. ${ }^{33}$ The investigators show that: Gal-3 protein expression is increased in a dosedependent manner on aldosterone treatment in cultured rat vascular smooth muscle cells within 24 hours, overexpression of recombinant human Gal-3 induced a 1.6-fold increase of collagen type I (Coll-I) deposition in rat vascular smooth muscle cells, and aldosterone-induced Coll-I deposition can be blocked by either Gal-3 neutralizing small interfering RNA (siRNA) or chemical inhibitors of Gal-3 carbohydratebinding activity. Finally, the authors of that study confirmed these in vitro data by showing that aldosterone treatment increased aortic Gal-3 and Coll-I expression in wild-type (WT) mice, whereas Gal-3 knockout $\left(\mathrm{Gal}-3^{--}\right)$mice are protected against these alterations. Thus, there is ample experimental evidence from different cardiovascular disease models that Gal-3 might be a biomarker involved in fibrosis induction and myocardial remodeling. ${ }^{34}$

Besides this, the active role of Gal-3 in the development of tissue fibrosis is underlined by the additional observations made in Gal-3 $3^{-/-}$mice in different disease models. Recently, it has been shown that Gal-3 mRNA and protein are upregulated in a mouse model of progressive renal fibrosis. ${ }^{35}$ The same study demonstrated that depletion of Gal-3 protects against renal fibrosis in this disease model. Notably, in the mouse model of unilateral ureteric obstruction 


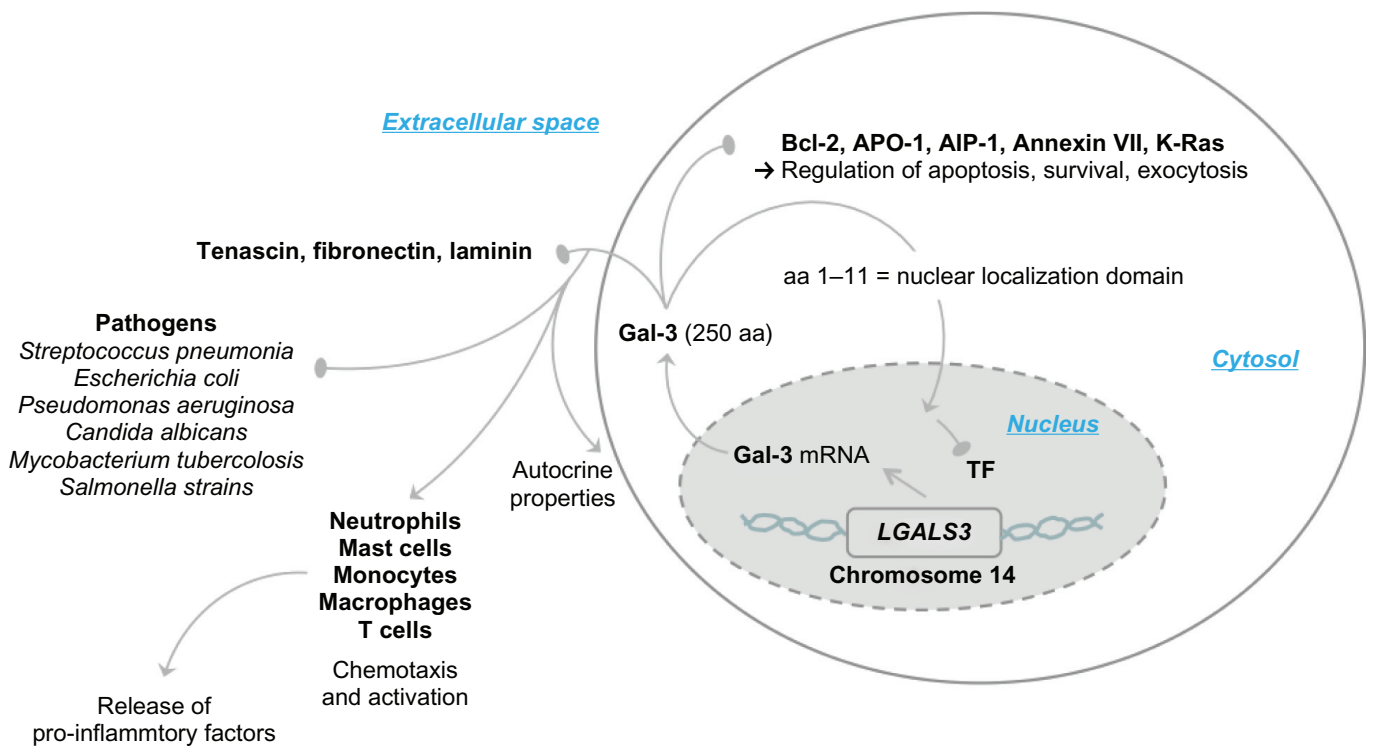

$\longrightarrow=$ Gal-3 binds to $\longrightarrow=$ Gal-3 translocates and/or interacts with

Figure I Overview about molecular background and function of Galectin-3.

Note: Chromosomal localization of human LGALS3 gene, potential localization of Galectin-3 protein and the biological properties of the lectin are depicted.

Abbreviations: aa, amino acid; TF, transcription factor; Bcl-2, B-cell lymphoma 2; APO-I, accumulation of photosystem I; AIP-I, actin interacting protein I; K-Ras, v-Ki-ras2

Kirsten rat sarcoma viral oncogene homolog.

(UUO) used, the depletion of macrophages also led to a significantly reduced activation of myofibroblasts, which are key players in fibrotic disorders, and reduced collagen expression. Importantly, the disruption of the Gal-3 gene affected neither macrophage recruitment to the affected kidney nor the secretion of pro-inflammatory cytokines from macrophages after interferon-gamma/lipopolysaccharide stimulation. In addition, the absence of endogenous Gal-3 did not alter transforming growth factor (TGF)- $\beta$ expression or TGF- $\beta$-receptor downstream signaling via Smad $2 / 3$. Considering these observations, the authors of that study concluded that Gal-3 secretion by macrophages is critical for the development of renal fibrosis, at least in the mouse model of UUO used.

Further evidence for the involvement of Gal-3 in the onset and progression of fibrotic disorders is supplied by work of the same research group performed on a carbon tetrachloride $\left(\mathrm{CCl}_{4}\right)$-induced liver injury model in mice. ${ }^{31}$ Henderson and colleagues showed that disruption of the Gal-3 gene inhibits myofibroblast activation and pro-collagen I expression though all investigated indicators of $\mathrm{CCl}_{4}$-induced liver injury, such as inflammation, and TGF- $\beta$ expression showed no difference between $\mathrm{Gal}^{-3^{--}}$and WT mice. Application of Gal-3 siRNA in WT mice on $\mathrm{CCl}_{4}$-induced liver injury supported the key role of Gal-3 in induction and progression of liver fibrosis.
The effect of Gal-3 disruption on different disease-specific readouts has been assessed in various disease models such as atherosclerosis, ischemic brain injury, during helminthic infection, in acetaminophen-induced hepatotoxicity, asthma, and in different infectious diseases. ${ }^{36-44}$

\section{Gal-3 in cardiovascular disease}

In recent years, a growing number of clinical studies examining the utility of Gal-3 as a biomarker for the management of cardiovascular disease have been published. The main findings from these studies are summarized in the following.

Clinical trials evaluating Gal-3 in cardiovascular disease patients can be categorized into four groups: those (1) assessing utility of the serum/plasma concentration of the lectin for diagnosis, (2) studying stratification for therapy, (3) monitoring therapy response, and (4) predicting short- and long-term morbidity and mortality.

\section{Diagnosis}

In 2006, van Kimmenade et a $1^{45}$ evaluated the utility of Gal-3 for diagnosis of HF in 599 patients of the previously reported N-terminal Pro-BNP Investigation of Dyspnea in the Emergency Department (PRIDE) study. ${ }^{46}$ Gal-3 was not correlated to the New York Heart Association (NYHA) Functional Classification and it also showed lower specificity and sensitivity 
in identifying HF within the cohort than amino terminal probrain natriuretic peptide (NT-proBNP) (receiver operating characteristic [ROC] curve analysis for NT-proBNP and Gal-3: 0.94, $P<0.0001$ and $0.72, P<0.0001$, respectively). The optimal cutoff for $80 \%$ specificity and $52 \%$ sensitivity was $6.88 \mathrm{ng}$ Gal-3/mL. The area under the curve (AUC) difference between NT-proBNP and Gal-3 was highly significant $(P<0.0001)$. Furthermore, there was no significant difference in median concentration of Gal-3 between ischemic and nonischemic HF patients or between patients displaying systolic versus non-systolic disease. Although Gal-3 did not outperform NT-proBNP in the diagnosis of HF, the assessment of this biomarker can complement the information gained by the natriuretic peptide. In any case, Gal-3 has the potential to predict outcomes for HF patients (see "Prediction of clinical outcome" later in this paper).

\section{Patient stratification}

Recently, plasma Gal-3 concentrations were assessed in patients with chronic HF upon treatment with rosuvastatin in a subpopulation of the Controlled Rosuvastatin Multinational Trial in Heart Failure (CORONA) trial. ${ }^{47}$ The purpose of this investigation was to assess whether patients with ischemic systolic HF displaying lower plasma Gal-3 levels benefit from statin therapy. Therefore, Gal-3 concentration was evaluated in 1492 patients, of whom 411 suffered nonfatal stroke, myocardial infarction, or cardiovascular death during a medium follow-up of approximately 33 months. Although rosuvastatin did not show a significant effect on primary endpoints (see Kjekshus et $\mathrm{l}^{48}$ for overall results of CORONA), subjects with plasma Gal-3 levels less than or equal to the median receiving statin therapy had a $30.4 \%$ lower event rate compared with placebo-treated patients of the same group $(P=0.019) .{ }^{48}$ Interestingly, patients with Gal-3 levels less than or equal to the median and NT-proBNP levels below $102.7 \mathrm{pmol} / \mathrm{L}$ exhibited particularly low cardiovascular event rates on rosuvastatin therapy (hazard ratio 0.33 ; confidence interval $0.16-0.67 ; P=0.002$ ). Conversely, no benefit of rosuvastatin therapy was observed in patients with Gal-3 levels greater than the median.

In a sub-study including approximately $30 \%$ of patients recruited to the large Valsartan Heart Failure Trial (Val-HeFT), the response to valsartan treatment was evaluated in patients displaying Gal-3 levels above or below the median. ${ }^{49-51}$ As with rosuvastatin in the CORONA trial, valsartan significantly reduced hospitalization for HF in patients with low baseline Gal-3 levels. In contrast, patients with Gal-3 levels above the median did not benefit from valsartan treatment.
Although the data generated in the CORONA and ValHeFT trial and the conclusions drawn from the results are of importance to further understand the usefulness of Gal-3 as a biomarker of cardiovascular disease, these findings, at least currently, have no implications for clinical practice.

A further trial to investigate whether Gal-3 levels can be applied to select patients for a certain therapy was performed by Stein. In a Multicenter Automatic Defibrillator Implantation with Cardiac Resynchronization Therapy (MADIT-CRT) sub-study, the investigators examined whether early stage HF patients could be selected and prioritized for cardiac resynchronization therapy (CRT) based on plasma Gal-3 concentrations. ${ }^{52}$ The study revealed that all patients benefited from the CRT regardless of their Gal-3 levels. However, patients displaying high Gal-3 levels benefited more from CRT, which is not surprising since higher Gal-3 levels mirror higher disease stage and worse prognosis (see "Prediction of clinical outcome"). Further studies in CRT patients are required to verify Stein's hypothesis that Gal-3 might be used to prioritize patients for this kind of intervention.

Stein's findings are in line with the recent findings in CRT patients in the Cardiac Resynchronization in Heart Failure (CARE-HF) study. ${ }^{53}$ Patients clearly benefited from CRT, as therapy was associated with improved outcome, which was defined as survival, development of worsening HF, leftventricular ejection fraction $(\mathrm{LVEF})>35 \%$, and NT-proBNP levels $<1000 \mathrm{pg} / \mathrm{mL}$. However, baseline Gal-3 concentrations did not predict response to CRT.

\section{Therapy monitoring}

The ability to detect beneficial effects of a (pharmaco)therapy on indices of cardiovascular disease early is important not only to the physician in their daily practice but also to study teams investigating effects of potential new chemical or biological entities. A biomarker indicating the modulation of pathomechanisms by an active substance on the molecular level before functional parameters of the heart change - such as ejection fraction (EF) or global longitudinal strain - has the potential to accelerate the development and approval of new drugs and may help to optimize management of cardiovascular disease.

To assess the utility of Gal-3 as a response-to-treatment biomarker, the longitudinal assessment of this factor on (pharmaco)therapy is key. Unfortunately, most published studies have evaluated Gal-3 concentrations only at baseline. However, the ability of Gal-3 to predict response to treatment was investigated in terminal HF patients needing mechanical circulatory support. ${ }^{54}$ In 55 patients with deteriorating 
HF, Gal-3 was measured pre- and postimplantation of a left-ventricular assist device (LVAD), total artificial heart (TAH), or heart and compared with levels in apparently healthy blood donor controls (mean $4.07 \mathrm{ng} / \mathrm{mL}$ ). Gal-3 was significantly increased in HF patients (mean $11 \mathrm{ng} / \mathrm{mL}$ ). Although there is ample experimental evidence indicating that Gal-3 is involved in development of fibrosis, mechanical unloading of the heart $(n=40)$ or removal of the failing ventricles $(n=15)$ did not reduce the plasma levels of Gal-3. ${ }^{29,31,32,54}$ However, patients who did not survive LVAD support had significantly elevated plasma concentrations of Gal-3 at the time of LVAD implantation compared with those patients who could be bridged to transplantation. ${ }^{54}$ These data were recently confirmed by the same group of investigators in a larger cohort of 151 ventricular assist device (VAD) patients. ${ }^{55}$ The ROC curve analysis for death under VAD support provided comparable data to previous reports with an AUC for Gal-3 of $0.64(P=0.009)$.

These data have raised the question of whether plasma Gal-3 might be a useful biomarker for prediction of mortality in patients with severe HF needing VAD, TAH, or heart transplant. Therefore, very recently, Erkilet et al investigated plasma Gal-3 levels as well as myocardial Gal-3 mRNA and protein expression in advanced HF patients needing VAD support. ${ }^{56}$ Plasma Gal-3 concentrations were significantly elevated in the group of 175 deteriorating HF patients compared with the 98 blood donor controls. Consistent with previous findings ${ }^{54}$ unloading of the heart did not influence plasma Gal-3 levels within the first 30 days after device implantation. Gal-3 mRNA and protein were detectable in the failing myocardium, but, interestingly, these did not correlate with its plasma concentration. This raises the question as to whether the failing myocardium is the primary source of elevated plasma Gal-3. Finally, Gal-3 did not provide sufficient discrimination for prediction of outcome after VAD implantation.

In the CARE-HF study, response of Gal-3 to CRT was evaluated in $260 \mathrm{HF}$ patients with NYHA class III or IV. ${ }^{53}$ Although patients benefited from CRT, no significant effect of therapy on Gal-3 levels was recorded within 18 months. In contrast, the pressure/volume overload biomarker NTproBNP mirrored positive effects of resynchronization.

The observation that Gal-3 does not mirror therapy response, made in the two aforementioned studies, was confirmed recently in the Deventer-Alkmaar heart failure (DEAL-HF) study. ${ }^{57}$ In 182 NYHA class III and IV HF patients there was no correlation between change in Gal-3 levels over time ( 3 months to baseline and 1 year to baseline) and change in left-ventricular end-diastolic volume, a measure of left-ventricular remodeling.

Considering these observations, it becomes clear there is no evidence to date that either serum or plasma Gal-3 concentration is a useful tool with which to monitor therapy response.

\section{Prediction of clinical outcome} Short-term outcome

The utility of Gal-3 in predicting clinical outcome was assessed, for example, in patients with acute dyspnea presenting to the emergency department in the PRIDE study. ${ }^{47}$ As already mentioned, Gal-3 concentration was found to be significantly increased in the plasma of HF patients in this study. During the follow-up at 60 days, 29\% of the 209 acute $\mathrm{HF}$ patients showed recurrent $\mathrm{HF}$ and $8 \%$ died. Median Gal-3 levels were significantly higher in patients who had recurrent HF or died within 60 days. The AUC of a ROC curve for the 60-day mortality or recurrent HF for Gal-3 was 0.74 $(P<0.0001)$ versus $0.67(P<0.009)$ for NT-proBNP. Based on this, the authors defined the cutoff for the prediction of 60-day mortality as $9.42 \mathrm{ng} / \mathrm{mL}$ Gal-3 (75\% sensitivity, $56 \%$ specificity). The adjusted multivariate analysis revealed that Gal-3 was superior to NT-proBNP. The rate of death or recurrent $\mathrm{HF}$ was highest in patients with combined elevated Gal-3 and NT-proBNP (>5562 pg/mL). Thus, although NTproBNP was superior in diagnosing HF in this study, Gal-3 was a stronger predictor of short-term mortality.

\section{Mid-term outcome}

The first evidence for a prognostic value of Gal-3 in patients with chronic HF came from the Coordinating study evaluating Outcomes of Advising and Counseling in Heart failure (COACH) study in which 592 patients with mild, moderate, and severe forms of the disease were investigated during a mean follow-up of 18 months. ${ }^{58}$ In 2011, the same group published an extended data package generated from this trial. ${ }^{59}$ The more recent publication includes data analysis of findings from the study of patients assigned to one of two groups: heart failure with reduced ejection fraction (HFREF) and heart failure with preserved ejection fraction (HFPEF). Individuals with a LVEF $>40 \%$ were assigned to the HFPEF group. The primary outcome measure of this study was a composite of all-cause mortality and HF hospitalization. This is one of the few studies so far that has analyzed Gal-3 not only at baseline but also in approximately $50 \%$ of patients after 6 months. The hazard ratio to reach the primary outcome increased constantly from the first to the fourth serum 
Gal-3 quartile. Changes of serum Gal-3 levels during first 6 months of the study did not add prognostic information to the baseline value. However, interestingly, while absolute Gal-3 levels did not differ between HFPEF and HFREF patients, the prognostic value of Gal-3 was more pronounced in HFPEF patients than in individuals with reduced EF. It was found that the same increase of Gal-3 levels in patients with HFPEF and HFREF was associated with a significantly stronger risk for reaching the primary outcome in patients with HFPEF. Taken together, these results show that baseline serum Gal-3 concentration has independent prognostic value for the endpoint all-cause mortality and HF hospitalization, even when corrected for established risk factors.

The predictive power of serum Gal-3 concentration was also assessed in HF patients (NYHA class III or IV) with evidence of left-ventricular systolic dysfunction and cardiac dyssynchrony as indicated by electrocardiogram or echocardiography in the CARE-HF study. ${ }^{53}$ Gal-3 was determined in serum at baseline and after 3 and 18 months. In this study, baseline Gal-3 was associated with death or HF hospitalization. The odds ratio (OR) for these endpoints in patients displaying a Gal-3 level of $>30 \mathrm{ng} / \mathrm{mL}$ was 2.05 .

The ability of Gal-3 to predict development of HF in patients suffering from acute coronary syndrome (ACS) was evaluated in a subgroup of the Pravastatin or Atorvastatin Evaluation and Infection Therapy - Thrombolysis In Myocardial Infarction 22 (PROVE-IT TIMI 22) trial. ${ }^{60}$ In this trial, 100 ACS patients were followed up for a mean duration of 2 years for the development of HF. Serum Gal-3 levels were increased in individuals who developed HF. Assessment of the unadjusted OR revealed a graded relationship between Gal-3 and the risk to develop HF. Patients in the second Gal-3 quartile (13.0-15.5 ng/mL) had an OR of 2 , whereas patients in the fourth quartile $(>19.2 \mathrm{ng} / \mathrm{mL})$ had an OR of 3.9.

A subgroup analysis of the Heart Failure: A Controlled Trial Investigating Outcomes of Exercise TraiNing (HFACTION) investigated the relationship between baseline plasma GAL-3 concentrations and all-cause hospitalization and all-cause mortality during a mean follow-up of 2.5 years in patients with chronic $\mathrm{HF}$ with an $\mathrm{EF}<35 \%{ }^{61}$ The investigators found that Gal-3 was associated with higher NYHA class, higher creatinine, lower maximal oxygen consumption, and lower systolic blood pressure. Interestingly, in this study, there was only a modest correlation between NT-proBNP and Gal-3 levels. When grouping patients according to whether they were below or above medium level for each biomarker, there was discordance between both biomarkers in $36 \%$ of patients $(292 / 815)$. However, there was a progressively increased hazard for low NT-proBNP/high Gal-3, high NT-proBNP/low Gal-3, and high NT-proBNP/high Gal-3 with regard to hospitalization-free survival.

\section{Long-term outcome}

The predictive value of Gal-3 was also analyzed in the recently published DEAL-HF study of 232 patients with chronic HF (NYHA class III-IV). ${ }^{62}$ During the follow-up of 6.5 years, $42 \%$ of recruited patients died. Approximately $50 \%$ of patients displayed a Gal-3 level above the upper normal level of $17.7 \mathrm{ng} / \mathrm{mL}$. Plasma Gal-3 concentrations were significantly correlated with age and NT-proBNP levels. Interestingly, there was no correlation between EF or type of HF and Gal-3. Patients who died during the follow-up period exhibited significantly higher baseline Gal-3 levels than the survivors. By ROC curve analysis, the authors found an AUC of $0.612(P=0.004)$ for Gal-3 for mortality within the study population, which was comparable to NT-proBNP (AUC 0.611 ). The highest product of sensitivity and specificity was found with $17.72 \mathrm{ng}$ Gal-3/mL. Even after adjustment for the covariates age, sex, estimated glomerular filtration rate, and NT-proBNP, plasma concentration of Gal-3 remained a strong predictor of mortality.

A second publication presenting data from an extended follow-up of $8.7 \pm 1$ year confirmed and expanded the initial findings. ${ }^{57}$ Kaplan-Meier analysis revealed that Gal-3 was associated with mortality during the follow-up period. Mortality rate increased across the Gal-3 quartiles. Fortunately and in contrast to most other trials assessing Gal-3, in the DEAL-HF program, serum samples were acquired not just at baseline but also after 3 and 12 months. When assessing the longitudinal course of Gal-3 levels, the investigators found that a change in Gal-3 concentration was not associated with survival and did not predict change of the left-ventricular end-diastolic volume. Therefore, the authors concluded that serial measurement does not add any benefit to single-point assessment. It is of note that Gal-3 plasma concentrations were significantly associated with renal dysfunction, which is of relevance because HF is often accompanied by impaired renal function. This finding is in line with the most recent findings of Erkilet and colleagues $^{56}$ and the results of the $\mathrm{COACH}$ study ${ }^{59}$ which underlines that Gal-3 is not a heart-specific biomarker. As such, conclusions drawn from Gal-3 serum levels must always be considered in the context of comorbidities, as some of the prognostic power of this biomarker may be mediated by renal function. Although the authors of the 
DEAL-HF study postulate that Gal-3 may potentially be used in the management of HF patients, the group provides no clear recommendation for the application of Gal-3 in clinical practice. ${ }^{62}$

Shah et al recently published data from 115 acutely decompensated HF patients, a subpopulation of the PRIDE study. ${ }^{45,63}$ Notably, Gal-3 was the strongest predictor of 4-year mortality when compared by multivariate analysis including echocardiographic indices. Patients with Gal-3 levels $>14.97 \mathrm{ng} / \mathrm{mL}$ had a hazard ratio of 5.5.

Finally, the relationship between Gal-3 and risk factors for cardiovascular disease as well as the predictive power of the lectin for cardiovascular and all-cause mortality were investigated in a large cohort of the Prevention of REnal and Vascular END stage disease (PREVEND) trial. ${ }^{64}$ The study recruited 7968 Caucasian individuals from the general population who were followed up for approximately 10 years, making this the largest study with the longest observation period investigating Gal-3 levels to date. Plasma Gal-3 concentrations were assessed at entry. The study found that Gal-3 was correlated with age; median Gal-3 levels were higher in women; Gal-3 was correlated with various cardiovascular risk factors such as body mass index, blood pressure, serum lipids, and renal function - this correlation was more pronounced in females; and baseline Gal-3 was an independent predictor of all-cause mortality in the general population but not of cancer or cardiovascular mortality.

A brief description of each of the clinical trials summarized in the present paper is presented in Table 1.

\section{Discussion and future directions}

"Biomarker" was defined by the National Institutes of Health in 2001 as "a characteristic that is objectively measured and evaluated as an indicator of normal biological process, pathogenic process or pharmacological response to a therapeutic intervention." 65 The appraisal of biomarker data can help address various challenges, such as early diagnosis of a disease, decision on the therapy most likely to be efficacious (personalized medicine), monitoring of response to intervention, exclusion of patients most likely to show unintended therapeutic side effects, and prediction of patient outcome in both clinical trials and daily clinical practice. Further, biomarkers are valuable tools supporting preclinical development through their use as indicators for efficacy or toxic/unintended effects of new chemical/biological entities in preclinical disease models. Biomarkers that behave similarly in animal models and human patients also have the potential to become bridging tools between research and clinical development.
Based on its utility in these applications, the value of Gal-3 as a biomarker in HF can be assessed.

\section{Support for decision-making in preclinical development}

As Gal-3 was found to be strongly upregulated in a rat model of renin-dependent hypertension - especially in those animals that progressed to $\mathrm{HF}^{35}$ - the lectin might be a valuable disease and response-to-treatment biomarker in HF animal models. However, more data from other species, especially on treatment with already-approved drugs, are desirable.

\section{Early diagnosis of disease}

The capability of Gal-3 to indicate HF seems to be limited. Kimmenade et al showed in patients with dyspnea that Gal-3 had a lower specificity and sensitivity than NT-proBNP to detect HF. ${ }^{46}$ Furthermore, Gal-3 did not correlate with NYHA class nor enable distinction between ischemic and nonischemic HF or patients displaying systolic or nonsystolic disease.

\section{Decision on the most efficacious therapy (personalized medicine/patient selection)}

Based on data published thus far, the utility of Gal-3 in patient/therapy selection cannot be assessed yet. Although response to treatment in patients displaying a baseline Gal-3 level below or above the mean has been assessed in statin, sartan, and cardiac resynchronization therapy, there still is no clear evidence that patients selected based on plasma Gal-3 levels are more likely to benefit from a certain therapy.

\section{Monitoring of response to intervention}

To date, there is no clear evidence that serum or plasma Gal-3 levels are useful in monitoring therapy response. This is underscored by the observation that mechanical unloading of the failing ventricle by VAD did not reduce serum Gal-3 concentrations. ${ }^{54-56}$ Even in patients clearly benefiting from CRT, no significant reduction of Gal-3 levels was observed within 18 months. ${ }^{53}$ Furthermore, in NYHA class III and IV patients, no correlation was seen between changes in echocardiographic indices of left-ventricular remodeling and longitudinal Gal-3 levels. ${ }^{57}$

\section{Exclusion of patients most likely to show unintended therapeutic side effects}

As far as this author is aware, no studies addressing this issue have been performed so far. 
Table I Clinical trials assessing Galectin-3

\begin{tabular}{|c|c|c|c|c|c|c|}
\hline Study & Patient population & Study focus & Follow-up & $\begin{array}{l}\text { Patients, } \\
\text { N }\end{array}$ & $\begin{array}{l}\text { Gal-3 } \\
\text { sampling }\end{array}$ & Reference(s) \\
\hline PRIDE & Acute dyspnea & $\begin{array}{l}\text { Diagnosis of } \mathrm{HF} \text { and } \\
\text { short-term prognosis }\end{array}$ & $60 d$ & 599 & BL & $\begin{array}{l}\text { van Kimmenade } \\
\text { et al }{ }^{45}\end{array}$ \\
\hline PRIDE & Acute dyspnea & $\begin{array}{l}\text { Relationship between Gal-3 and } \\
\text { cardiac structure (echocardiography) }\end{array}$ & $4 y$ & 115 & $\mathrm{BL}$ & Shah et $\mathrm{al}^{63}$ \\
\hline CORONA & $\begin{array}{l}\text { Ischemic systolic } \\
\text { HF }\end{array}$ & $\begin{array}{l}\text { Utility of Gal-3 to predict response } \\
\text { to rosuvastatin therapy }\end{array}$ & $32.8 \mathrm{mth}$ & 1492 & $\mathrm{BL}$ & Gullestad et al ${ }^{47}$ \\
\hline CARE-HF & $\begin{array}{l}\text { LV dysfunction and } \\
\text { dyssynchrony }\end{array}$ & $\begin{array}{l}\text { Association of Gal-3 with long-term } \\
\text { CV outcome upon CRT }\end{array}$ & $18 \mathrm{mth}$ & 260 & $\begin{array}{l}\text { BL, } 3 \text { mth, } \\
18 \text { mth }\end{array}$ & $\begin{array}{l}\text { Lopez-Andrès } \\
\text { et al }{ }^{53}\end{array}$ \\
\hline $\mathrm{COACH}$ & HFPEF and HFREF & $\begin{array}{l}\text { Predictive value of Gal-3 in HFPEF } \\
\text { and HFREF }\end{array}$ & $18 \mathrm{mth}$ & 592 & $\mathrm{BL}$ & $\begin{array}{l}\text { de Boer } \\
\text { et al }{ }^{59}\end{array}$ \\
\hline MADIT-CRT & $\begin{array}{l}\text { Nonischemic early } \\
\text { stage HF (EF < 30\%) }\end{array}$ & $\begin{array}{l}\text { Utility of Gal-3 to prioritize patients } \\
\text { for CRT }\end{array}$ & $2.4 y$ & $|76|$ & $\mathrm{BL}$ & Stein ${ }^{52}$ \\
\hline $\begin{array}{l}\text { PROVE-IT } \\
\text { TIMI } 22\end{array}$ & $\begin{array}{l}\text { Unstable angina or } \\
\text { myocardial infarction }\end{array}$ & $\begin{array}{l}\text { Relationship between Gal-3 and } \\
\text { development of HF on ACS }\end{array}$ & $2 y$ & 100 & $B L$ & Grandin et al ${ }^{60}$ \\
\hline DEAL-HF & $\begin{array}{l}\text { Chronic HF (NYHA } \\
\text { class III-IV) }\end{array}$ & Prognostic value of Gal-3 & $6.5 y$ & 232 & BL & Lok et $\mathrm{al}^{62}$ \\
\hline DEAL-HF & $\begin{array}{l}\text { Chronic HF (NYHA } \\
\text { class III-IV) }\end{array}$ & $\begin{array}{l}\text { Utility of Gal-3 as marker of } \\
\text { LV remodeling and prognostic factor }\end{array}$ & $8.7 y$ & 240 & $\begin{array}{l}\text { BL, } 3 \text { mth, } \\
\text { I y }\end{array}$ & Lok et $\mathrm{al}^{57}$ \\
\hline Val-HeFT & $\mathrm{HF}(\mathrm{EF}<40 \%)$ & $\begin{array}{l}\text { Response to valsartan in patients } \\
\text { with Gal-3 above or below mean }\end{array}$ & $12 \mathrm{mth}$ & $\sim 1500$ & $\begin{array}{l}\text { BL, } 4 \text { mth, } \\
12 \mathrm{mth}\end{array}$ & Anand $\mathrm{I}^{51}$ \\
\hline PREVEND & $\begin{array}{l}\text { Caucasian general } \\
\text { population }\end{array}$ & $\begin{array}{l}\text { Association between Gal-3 with } \\
\mathrm{CV} \text { and general outcome }\end{array}$ & $10 y$ & 7968 & $\mathrm{BL}$ & de Boer et al ${ }^{64}$ \\
\hline HF-ACTION & $\begin{array}{l}\text { Chronic HF (NYHA } \\
\text { class II-IV), (EF }<35 \%)\end{array}$ & $\begin{array}{l}\text { Association between Gal-3 with all-cause } \\
\text { hospitalization/all-cause mortality }\end{array}$ & $2.5 \mathrm{y}$ & 895 & BL & Felker et $\mathrm{a}^{\mid 61}$ \\
\hline Not applicable & VAD, Htx, TAH & Response of Gal-3 to unloading of heart & $30 d$ & 55 & $\mathrm{BL}, 30 \mathrm{~d}$ & Milting et $\mathrm{al}^{54}$ \\
\hline Not applicable & VAD & $\begin{array}{l}\text { Predictive value of plasma Gal-3 for } \\
\text { death on device. Myocardial Gal-3 } \\
\text { messenger RNA and protein expression }\end{array}$ & Ongoing & 175 & $\mathrm{BL}, 30 \mathrm{~d}$ & Erkilet et al ${ }^{56}$ \\
\hline
\end{tabular}

Abbreviations: BL, baseline Gal-3 was determined at study entry; CARE-HF, Cardiac Resynchronization in Heart Failure; COACH, Coordinating study evaluating Outcomes of Advising and Counselling in Heart failure; CRT, cardiac resynchronization therapy; CORONA, Controlled Rosuvastatin Multinational Trial in Heart Failure; CV, cardiovascular; d, days; EF, ejection fraction; HF, heart failure; HF-ACTION, Heart Failure: A Controlled Trial Investigating Outcomes of Exercise TraiNing; HFREF, heart failure with reduced ejection fraction; HFPEF, heart failure with preserved ejection fraction; Htx, heart transplantation; L, longitudinal assessment of Gal-3; LV, left ventricular; MADIT-CRT, Multicenter Automatic Defibrillator Implantation with Cardiac Resynchronization Therapy; mth, months; NYHA, New York Heart Association; PRIDE, N-terminal Pro-BNP Investigation of Dyspnea in the Emergency Department; PROVE-IT TIMI 22, Pravastatin or Atorvastatin Evaluation and Infection Therapy Thrombolysis In Myocardial Infarction 22; TAH, total artificial heart; VAD, ventricular assist device; Val-HeFT, Valsartan Heart Failure Trial; $y$, years; PREVEND, Prevention of REnal and Vascular END stage disease; DEAL-HF, Deventer-Alkmaar heart failure.

\section{Prediction of patient outcome}

Most reported studies have investigated the predictive value of baseline Gal-3 levels. ${ }^{45,47,51,59,60,61-64}$ Strong evidence has accumulated that Gal-3 is a strong predictor of short- ${ }^{46}$ mid-, ${ }^{53,58-61}$ and long-term mortality ${ }^{45,57,62-64}$ as well as HFassociated hospitalization in different HF patient populations, including early ${ }^{51}$ and late-stage disease as well as HFPEF and HFREF. ${ }^{59}$ Furthermore, Gal-3 predicted development of HF after ACS. ${ }^{60}$

\section{General considerations}

Although the studies analyzed in this review are sub-studies of larger trials, more than 16,900 patients were characterized in terms of their Gal-3 levels in these investigations, which probably makes Gal-3 one of the best-described cardiovascular biomarker candidates after brain natriuretic peptide and NT-proBNP. Nevertheless, at present, it is not clear how to translate these results into clinical guidelines for patient management. Compared with oncology, in which the use of biomarkers - for example, for patient selection - is already part of daily practice, the application of biomarkers, such as Gal-3, in cardiology is still in its infancy. Furthermore, Gal-3 is not a heart-specific biomarker. The lectin is also involved - for instance, in the development of experimental renal disease (see "Support for decision-making in preclinical development") - and there are clear suggestions that Gal-3 levels are correlated with the severity of renal dysfunction in HF patients. ${ }^{63}$

Moreover, it is of note that Gal-3 also seems to be an important player in the development, growth, and metastasis of tumors. In contrast to the multiple studies that have investigated Gal-3 in cardiovascular diseases, little has 
been published so far on the utility of this biomarker in oncological indications. Although it is far beyond the scope of this manuscript to review the role of Gal-3 in oncology, it is worth noting that data recently published by de Boer et al showed that Gal-3 is a strong predictor of cancer mortality. ${ }^{64}$

\section{Conclusion}

A significant number of clinical studies investigating properties and utility of Gal-3 emphasis its role as a promising HF biomarker candidate. Nevertheless, more clinical studies investigating Gal-3 longitudinally, especially on therapy, are desirable. Future studies have to clearly demonstrate how individual Gal-3 levels can guide decision-making in daily clinical practice or during the drug-development process and thereby improve HF patient care.

\section{Acknowledgment}

The author thanks Patrick Brehm for editorial support.

\section{Disclosure}

The author is a full time employee of Bayer HealthCare AG, Germany and declares no other conflicts of interest in this work.

\section{References}

1. Ochieng J, Furtak V, Lukyanov P. Extracellular functions of galectin-3. Glycoconj J. 2004;19(7-9):527-535.

2. Raimond J, Zimonjic DB, Mignon C, et al. Mapping of the galectin-3 gene (LGALS3) to human chromosome 14 at region 14q21-22. Mamm Genome. 1997;8(9):706-707.

3. Kim H, Lee J, Hyun JW, Park JW, Joo HG, Shin T. Expression and immunohistochemical localization of galectin-3 in various mouse tissues. Cell Biol Int. 2007;31(7):655-662.

4. Gong HC, Honjo Y, Nangia-Makker P, et al. The NH2 terminus of galectin-3 governs cellular compartmentalization and functions in cancer cells. Cancer Res. 1999;59(24):6239-6245.

5. Hughes RC. Secretion of the galectin family of mammalian carbohydratebinding proteins. Biochim Biophys Acta. 1999;1473(1):172-185.

6. Lin HM, Pestell RG, Raz A, Kim HR. Galectin-3 enhances cyclin D(1) promoter activity through SP1 and a cAMP-responsive element in human breast epithelial cells. Oncogene. 2002;21(52):8001-8010.

7. Paron I, Scaloni A, Pines A, et al. Nuclear localization of Galectin-3 in transformed thyroid cells: a role in transcriptional regulation. Biochem Biophys Res Commun. 2003;302(3):545-553.

8. Dagher SF, Wang JL, Patterson RJ. Identification of galectin- 3 as a factor in pre-mRNA splicing. Proc Natl Acad Sci U SA. 1995;92(4):1213-1217.

9. Patterson RJ, Wang W, Wang JL. Understanding the biochemical activities of galectin-1 and galectin-3 in the nucleus. Glycoconj $J$. 2004;19(7-9):499-506.

10. Sato S, Burdett I, Hughes RC. Secretion of the baby hamster kidney $30-\mathrm{kDa}$ galactose-binding lectin from polarized and nonpolarized cells: a pathway independent of the endoplasmic reticulum-Golgi complex. Exp Cell Res. 1993;207(1):8-18.

11. Sato S, Hughes RC. Regulation of secretion and surface expression of Mac-2, a galactoside-binding protein of macrophages. J Biol Chem. 1994;269(6):4424-4430.
12. Mehul B, Hughes RC. Plasma membrane targetting, vesicular budding and release of galectin 3 from the cytoplasm of mammalian cells during secretion. J Cell Sci. 1997;110(Pt 10):1169-1178.

13. Théry C, Boussac M, Véron P, et al. Proteomic analysis of dendritic cell-derived exosomes: a secreted subcellular compartment distinct from apoptotic vesicles. J Immunol. 2001;166(12):7309-7318.

14. Ochieng J, Leite-Browning ML, Warfield P. Regulation of cellular adhesion to extracellular matrix proteins by galectin-3. Biochem Biophys Res Commun. 1998;246(3):788-791.

15. Dong S, Hughes RC. Macrophage surface glycoproteins binding to galectin-3 (Mac-2-antigen). Glycoconj J. 1997;14(2):267-274.

16. Hughes RC. Galectins as modulators of cell adhesion. Biochimie. 2001;83(7):667-676.

17. Sato S, Ouellet N, Pelletier I, Simard M, Rancourt A, Bergeron MG. Role of galectin-3 as an adhesion molecule for neutrophil extravasation during streptococcal pneumonia. J Immunol. 2002;168(4): $1813-1822$.

18. Swarte VV, Mebius RE, Joziasse DH, Van den Eijnden DH, Kraal G. Lymphocyte triggering via L-selectin leads to enhanced galectin-3-mediated binding to dendritic cells. Eur J Immunol. 1998;28(9):2864-2871.

19. Dumic J, Dabelic S, Flögel M. Galectin-3: an open-ended story. Biochim Biophys Acta. 2006;1760(4):616-635.

20. Colnot C, Ripoche MA, Milon G, Montagutelli X, Crocker PR, Poirier F. Maintenance of granulocyte numbers during acute peritonitis is defective in galectin-3-null mutant mice. Immunology. 1998; 94(3):290-296.

21. Hsu DK, Yang RY, Pan Z, et al. Targeted disruption of the galectin-3 gene results in attenuated peritoneal inflammatory responses. $\mathrm{Am} \mathrm{J}$ Pathol. 2000;156(3):1073-1083.

22. Sato S, Nieminen J. Seeing strangers or announcing "danger": galectin-3 in two models of innate immunity. Glycoconj J. 2004;19(7-9): 583-591.

23. Karlsson A, Christenson K, Matlak M, et al. Galectin-3 functions as an opsonin and enhances the macrophage clearance of apoptotic neutrophils. Glycobiology. 2009;19(1):16-20.

24. Nieminen J, St-Pierre C, Bhaumik P, Poirier F, Sato S. Role of galectin-3 in leukocyte recruitment in a murine model of lung infection by Streptococcus pneumoniae. J Immunol. 2008;180(4):2466-2473.

25. Mey A, Leffler H, Hmama Z, Normier G, Revillard JP. The animal lectin galectin-3 interacts with bacterial lipopolysaccharides via two independent sites. J Immunol. 1996;156(4):1572-1577.

26. Gupta SK, Masinick S, Garrett M, Hazlett LD. Pseudomonas aeruginosa lipopolysaccharide binds galectin-3 and other human corneal epithelial proteins. Infect Immun. 1997;65(7):2747-2753.

27. Kohatsu L, Hsu DK, Jegalian AG, Liu FT, Baum LG. Galectin-3 induces death of Candida species expressing specific beta-1,2-linked mannans. J Immunol. 2006;177(7):4718-4726.

28. Beatty WL, Rhoades ER, Hsu DK, Liu FT, Russell DG. Association of a macrophage galactoside-binding protein with Mycobacteriumcontaining phagosomes. Cell Microbiol. 2002;4(3):167-176.

29. Sharma UC, Pokharel S, van Brakel TJ, et al. Galectin-3 marks activated macrophages in failure-prone hypertrophied hearts and contributes to cardiac dysfunction. Circulation. 2004;110(19):3121-3128.

30. Schroen B, Heymans S, Sharma U, et al. Thrombospondin-2 is essential for myocardial matrix integrity: increased expression identifies failureprone cardiac hypertrophy. Circ Res. 2004;95(5):515-522.

31. Henderson NC, Mackinnon AC, Farnworth SL, et al. Galectin-3 regulates myofibroblast activation and hepatic fibrosis. Proc Natl Acad Sci USA. 2006;103(13):5060-5065.

32. Liu YH, D'Ambrosio M, Liao TD, et al. N-acetyl-seryl-aspartyllysyl-proline prevents cardiac remodeling and dysfunction induced by galectin-3, a mammalian adhesion/growth-regulatory lectin. Am J Physiol Heart Circ Physiol. 2009;296(2):H404-H412.

33. Calvier L, Miana M, Reboul P, et al. Galectin-3 mediates aldosterone-induced vascular fibrosis. Arterioscler Thromb Vasc Biol. 2013;33(1):67-75. 
34. de Boer RA, Yu L, van Veldhuisen DJ. Galectin-3 in cardiac remodeling and heart failure. Curr Heart Fail Rep. 2010;7(1):1-8.

35. Henderson NC, Mackinnon AC, Farnworth SL, et al. Galectin-3 expression and secretion links macrophages to the promotion of renal fibrosis. Am J Pathol. 2008;172(2):288-298.

36. Nachtigal M, Ghaffar A, Mayer EP. Galectin-3 gene inactivation reduces atherosclerotic lesions and adventitial inflammation in ApoE-deficient mice. Am J Pathol. 2008;172(1):247-255.

37. Doverhag C, Hedtjärn M, Poirier F, et al. Galectin-3 contributes to neonatal hypoxic-ischemic brain injury. Neurobiol Dis. 2010;38(1):36-46.

38. Breuilh L, Vanhoutte F, Fontaine J, et al. Galectin-3 modulates immune and inflammatory responses during helminthic infection: impact of galectin-3 deficiency on the functions of dendritic cells. Infect Immun. 2007;75(11):5148-5157.

39. Dragomir AC, Sun R, Mishin V, Hall LB, Laskin JD, Laskin DL. Role of galectin-3 in acetaminophen-induced hepatotoxicity and inflammatory mediator production. Toxicol Sci. 2012;127(2):609-619.

40. Zuberi RI, Hsu DK, Kalayci O, et al. Critical role for galectin-3 in airway inflammation and bronchial hyperresponsiveness in a murine model of asthma. Am J Pathol. 2004;165(6):2045-2053.

41. Oliveira FL, Brand C, Paula AA, et al. Lack of galectin-3 disturbs mesenteric lymph node homeostasis and B cell niches in the course of Schistosoma mansoni infection. PLoS One. 2011;6(5):e19216.

42. Ferraz LC, Bernardes ES, Oliveira AF, et al. Lack of galectin-3 alters the balance of innate immune cytokines and confers resistance to Rhodococcus equi infection. Eur J Immunol. 2008;38(10):2762-2775.

43. Silva-Monteiro E, Reis Lorenzato L, Kenji Nihei O, et al. Altered expression of galectin-3 induces cortical thymocyte depletion and premature exit of immature thymocytes during Trypanosoma cruzi infection. Am J Pathol. 2007;170(2):546-556.

44. Farnworth SL, Henderson NC, Mackinnon AC, et al. Galectin-3 reduces the severity of pneumococcal pneumonia by augmenting neutrophil function. Am J Pathol. 2008;172(2):395-405.

45. van Kimmenade RR, Januzzi JL Jr, Ellinor PT, et al. Utility of aminoterminal pro-brain natriuretic peptide, galectin-3, and apelin for the evaluation of patients with acute heart failure. $\mathrm{J} \mathrm{Am} \mathrm{Coll} \mathrm{Cardiol.}$ 2006;48(6):1217-1224.

46. Januzzi JL Jr, Camargo CA, Anwaruddin S, et al. The N-terminal ProBNP investigation of dyspnea in the emergency department (PRIDE) study. Am J Cardiol. 2005;95(8):948-954.

47. Gullestad L, Ueland T, Kjekshus J, et al; CORONA Study Group. Galectin-3 predicts response to statin therapy in the Controlled Rosuvastatin Multinational Trial in Heart Failure (CORONA). Eur Heart J. 2012;33(18):2290-2296.

48. Kjekshus J, Apetrei E, Barrios V, et al; CORONA Group. Rosuvastatin in older patients with systolic heart failure. $N$ Engl J Med. 2007; 357(22):2248-2261.

49. Cohn JN, Tognoni G, Glazer RD, Spormann D, Hester A. Rationale and design of the Valsartan Heart Failure Trial: a large multinational trial to assess the effects of valsartan, an angiotensin-receptor blocker, on morbidity and mortality in chronic congestive heart failure. $J$ Card Fail. 1999;5(2):155-160.

50. Cohn JN, Tognoni G; Valsartan Heart Failure Trial Investigators. A randomized trial of the angiotensin-receptor blocker valsartan in chronic heart failure. N Engl J Med. 2001;345(23):1667-1675.
51. Anand IS, Rector TS, Kuskowski M, Adourian A, Muntendam P, Cohn JN. Baseline and serial measurements of galectin-3 in patients with heart failure: relationship to prognosis and effect of treatment with valsartan in the Val-HeFT. Eur J of Heart Fail. Epub 2013 Jan 4.

52. Stein K. Galectin-3 in candidates for CRT treatment: a MADIT-CRT substudy. Paper presented at the American College of Cardiology 61st Annual Scientific Session and Expo; March 26, 2012; Chicago, IL.

53. Lopez-Andrès N, Rossignol P, Iraqi W, Fay R, Nuée J, Ghio S, et al. Association of galectin-3 and fibrosis markers with long-term cardiovascular outcomes in patients with heart failure, left ventricular dysfunction, and dyssynchrony: insights from the CARE-HF (Cardiac Resynchronization in Heart Failure) trial. Eur J Heart Fail. 2012;14(1): 74-81.

54. Milting H, Ellinghaus P, Seewald M, et al. Plasma biomarkers of myocardial fibrosis and remodeling in terminal heart failure patients supported by mechanical circulatory support devices. J Heart Lung Transplant. 2008;27(6):589-596.

55. Erkilet G, Schulte-Eistrup S, Morshuis M, et al. Plasma galectin 3 is increased in terminal heart failure patients and is elevated in patients not surviving mechanical circulatory support. J Heart Lung Transplant. 2010;29(2):S65

56. Erkilet G, Özpeker C, Böthig D, et al. The biomarker plasma galectin-3 in advanced heart failure and survival with mechanical circulatory support devices. J Heart Lung Transplant. 2013;32(2):221-230.

57. Lok DJ, Lok SI, Bruggink-André de la Porte PW, et al. Galectin-3 is an independent marker for ventricular remodeling and mortality in patients with chronic heart failure. Clin Res Cardiol. Epub August 12, 2012.

58. van Veldhuisen DJ, Lok DJ, Damman K, et al. Clinical and prognostic value of galectin-3, a novel fibrosis-associated biomarker, in patients with chronic heart failure. J Card Fail. 2009;15(9):841.

59. de Boer RA, Lok DJ, Jaarsma T, et al. Predictive value of plasma galectin-3 levels in heart failure with reduced and preserved ejection fraction. Ann Med. 2011;43(1):60-68.

60. Grandin EW, Jarolim P, Murphy SA, et al. Galectin-3 and the development of heart failure after acute coronary syndrome: pilot experience from PROVE IT-TIMI 22. Clin Chem. 2012;58(1):267-273.

61. Felker GM, Fiuzat M, Shaw LK, et al. Galectin-3 in ambulatory patients with heart failure: results from the HF-ACTION study. Circ Heart Fail. 2012;5(1):72-78.

62. Lok DJ, Van Der Meer P, de la Porte PW, et al. Prognostic value of galectin-3, a novel marker of fibrosis, in patients with chronic heart failure: data from the DEAL-HF study. Clin Res Cardiol. 2010; 99(5):323-328.

63. Shah RV, Chen-Tournoux AA, Picard MH, van Kimmenade RR, Januzzi JL. Galectin-3, cardiac structure and function, and long-term mortality in patients with acutely decompensated heart failure. Eur $J$ Heart Fail. 2010;12(8):826-832.

64. de Boer RA, van Veldhuisen DJ, Gansevoort RT, et al. The fibrosis marker galectin-3 and outcome in the general population. J Intern Med. 2012;272(1):55-64.

65. Biomarkers Definitions Working Group. Biomarkers and surrogate endpoints: preferred definitions and conceptual framework. Clin Pharmacol Ther. 2001;69(3):89-95.
Research Reports in Clinical Cardiology

\section{Publish your work in this journal}

Research Reports in Clinical Cardiology is an international, peerreviewed, open access journal publishing original research, reports, editorials, reviews and commentaries on all areas of cardiology in the clinic and laboratory. The manuscript management system is completely online and includes a very quick and fair peer-review system.

\section{Dovepress}

Visit http://www.dovepress.com/testimonials.php to read real quotes from published authors. 